PROCEEDINGS OF THE

AMERICAN MATHEMATICAL SOCIETY

Volume 129, Number 5, Pages 1525-1534

S 0002-9939(01)05984-6

Article electronically published on January 8, 2001

\title{
THE ACTION OF A SEMISIMPLE LIE GROUP ON ITS MAXIMAL COMPACT SUBGROUP
}

\author{
T. BUDAK, N. IŞIK, P. MILNES, AND J. PYM \\ (Communicated by Michael Handel)
}

\begin{abstract}
In this paper we determine the structure of the minimal ideal in the enveloping semigroup for the natural action of a connected semisimple Lie group on its maximal compact subgroup. In particular, if $G=K A N$ is an Iwasawa decomposition of the group $G$, then the group in the minimal left ideal is isomorphic both algebraically and topologically with the normalizer $M$ of $A N$ in $K$. Complete descriptions are given for the enveloping semigroups in the cases $G=\mathrm{SL}(2, \mathbb{C})$ and $G=\mathrm{SL}(2, \mathbb{R})$.
\end{abstract}

\section{INTRODUCTION}

Throughout this paper $G$ will be a connected semisimple Lie group with finite centre. It therefore has an Iwasawa decomposition, that is $G=K A N$ is a product of closed connected subgroups with $K$ compact, $A$ abelian and $N$ nilpotent, and the decomposition $g=k a n$ of any element of $G$ is unique. The prime examples of these groups are the special linear groups $\operatorname{SL}(n, \mathbb{R})$ and $\operatorname{SL}(n, \mathbb{C})$; here a suitable decomposition can be obtained by taking $K=\mathrm{SO}(n, \mathbb{R})$ in the real case and $\mathrm{SU}(n)$ in the complex case, $A$ to be the diagonal matrices with positive entries and determinant 1 , and $N$ to be the upper triangular matrices with 1 's on the diagonal.

The subgroup $A N$ is closed and the homogeneous space $G / A N$ is naturally homeomorphic to the compact space $K$. $G$ therefore has a natural action on $K$; if we identify the coset $g A N$ with $k \in K$ we can indicate the action of $g^{\prime} \in G$ by

$$
g^{\prime}: k=g A N \mapsto g^{\prime}(k)=g^{\prime} g A N .
$$

The restriction of this action to $K \subset G$ is just left multiplication by members of $K$, that is, $k^{\prime}(k)=k^{\prime} k$. Thus these flows $(G, K)$ are not only minimal (each orbit is dense) but transitive (given $k, k^{\prime} \in K$ we can find $g \in G$ with $g k=k^{\prime}$ ).

In general, for any flow $(H, X)$ consisting of a semigroup $H$ acting continuously on a compact space $X$ we can regard each $h \in H$ as a continuous mapping $X \rightarrow X$ and then the closure of $H$ in the compact semigroup $X^{X}$ of all maps from $X$ to itself is a subsemigroup $\Sigma=\Sigma(H, X)$ which is compact and is called the enveloping semigroup of the flow. Multiplication is continuous in $\Sigma$ in the left-hand variable $(x \mapsto x y$ is continuous for each $y)$. The structure theory for such semigroups ([2], Theorems 1.3 .11 and 1.2.16) tells us that $\Sigma$ has minimal left ideals $L=L_{\Sigma}$. Any

Received by the editors July 15, 1999.

2000 Mathematics Subject Classification. Primary 54H15, 54H20, 57S20.

The first and second authors were supported by a research grant from Boğaziçi University.

The third author was supported by NSERC grant A7857. 
two $L$ 's are isomorphic both topologically and algebraically. Each is compact. The set $E=E_{\Sigma}$ of idempotents in $L$ is a left zero semigroup $\left(e e^{\prime}=e\right.$ for all $\left.e, e^{\prime}\right), L$ has maximal subgroups $\Gamma=\Gamma_{\Sigma}$ (all algebraically isomorphic), and algebraically $L$ is the direct product $E \times \Gamma$. If the flow $(H, X)$ is minimal, then $L x=X$ for each $x \in X$ ([2], Proposition 1.6.12). This shows that for minimal flows the action of $\Sigma$ on $X$ is determined by the semigroup product $\Sigma \times L \rightarrow L$. Thus knowledge of a minimal left ideal plays a key role in the analysis of flows.

In this paper we shall determine $L$ for the action of $G$ on $G / A N \cong K$. To be precise:

Theorem 1.1. Let $G$ be a connected semisimple Lie group with finite centre and Iwasawa decomposition $G=K A N$. Then the minimal left ideal $L_{\Sigma(G, K)}$ is homeomorphic with $K$. If $M$ is the normaliser of $A N$ in $K$, then the group $\Gamma_{\Sigma(G, K)}$ is isomorphic (algebraically and topologically) with $M$ and the cardinal of the semigroup $E_{\Sigma(G, K)}$ of idempotents in $L_{\Sigma(G, K)}$ is the cardinal of $K / M$.

Flows which are isomorphic with the flow on a minimal left ideal of the enveloping semigroup are discussed in Theorem IV(3.44) of [10.

This result has similarities with Theorem 9 of the paper [8]. There the group $G$ was embedded in its largest semigroup compactification $G^{\mathcal{L U C}}$. For each compact normal subgroup $N$ of $G$ and each idempotent $e \in G^{\mathcal{L U C}}$ it was found that $N e$ had the algebraic structure $E \times \Gamma$ as above, where $E$ was a left zero semigroup and $\Gamma$ a group; moreover $\Gamma=e N e$ and $E=\{n e: n \in N$ and $n e=e n e\}$. Theorem 1.1 is superficially the same. If $e$ is a minimal idempotent in $\Sigma$, then $K e=L$ is algebraically $E \times \Gamma, e K e=\Gamma=M$ and $E=\{k e: k \in K$ and $k e=e k e\}$. However, here we do not have the conclusion except when the idempotent $e$ is minimal, and the further conclusion in [8] that $N$ can be written as a semidirect product does not hold. The difference here is that $K$ is not normal; indeed $G$ is only a Zappa product of $K$ and $A N$.

It is often hard to obtain detailed information about semigroup compactifications of a group $G$, and in particular about the decomposition of a minimal left ideal in the form $L=E \times \Gamma$. One particular compactification which has been much studied is the largest semigroup compactification $G^{\mathcal{L U C}}$ ('largest' meaning that every other compactification is a continuous homomorphic image of $G^{\mathcal{L U C}}$ ). One natural question is whether the semigroup $E$ of idempotents is closed for $G^{\mathcal{L U C}}$. This was shown not to be so in the case $G=\mathbb{Z}$ by J. Auslander (unpublished). We shall obtain this result for groups in our class when $M$ is finite, and also for the group $\mathrm{SL}(2, \mathbb{C})$ for which $M$ is infinite. Another question is how big the group $\Gamma$ must be, and indeed whether it must be non-trivial. The latter question appears to be open in the case in which $G$ is minimally almost periodic (that is, the largest topological group compactification of $G$ is just a singleton). Since the $\operatorname{groups} \operatorname{SL}(n, \mathbb{R})$ are minimally almost periodic, it is of interest that our present approach gives a little more information. We need a simple result: a surjective homomorphism from one compact semigroup onto another sends a maximal subgroup in a minimal left ideal of the first onto a corresponding object in the second (a proof can be found in Lemma 2.3 of [7]). Theorem 1.1 then yields immediately

Corollary 1.2. Let $G$ be as in Theorem 1.1 and let $\Gamma$ be the maximal subgroup in a minimal left ideal of $G^{\mathcal{L U C}}$. Then $M$ is a homomorphic image of $\Gamma$. 
Illustrations of the complexity of $M$ can be found in [6], p. 324 and also Chap. VII, especially $\S 5$, and [11], pp. 28-29. For the examples $\operatorname{SL}(2, \mathbb{R})$ and $\operatorname{SL}(2, \mathbb{C})$ considered in $\S 3, M=\{ \pm 1\}$ and $\mathbb{T}$ respectively.

In $\S 3$ we illustrate the general theory by looking at the particular groups $\operatorname{SL}(2, \mathbb{C})$ and $\operatorname{SL}(2, \mathbb{R})$ in more detail. We find that the enveloping semigroups are remarkably small here.

\section{The Proof of Theorem 1.1}

To prove Theorem 1.1 we need to consider some flows related to $(G, G / A N \cong$ $K$ ). The product $A N$ is a closed subgroup of $G$ and is isomorphic (algebraically and topologically) to the semi-direct product of $A$ and $N$, with $N$ normal in $A N$. Since $A N \subset G$, we have $\Sigma(A N, G / A N) \subset \Sigma(G, G / A N)$. Again, $M A N$ is a closed subgroup of $G$; it is the normalizer of $N$ in $G$. We can therefore consider the flows $(G, G / M A N)$ and $(A N, G / M A N)$. The flow $(G, G / M A N)$ has been studied by many others (see Glasner [4, Warner [11) with other aims in view. The key result for us is due to Moore 9] (see also de Vries [10, $\mathrm{VI}(6.1) 5$ and $\mathrm{VI}(1.8)$ ); in the language of flows it implies that

Theorem 2.1. The flow $(G, G / M A N)$ is proximal.

The meaning of proximal is that, for any pair $x, y \in G / M A N$, there is a net $\left(g_{i}\right)$ in $G$ such that $\lim _{i} g_{i}(x)=\lim _{i} g_{i}(y)$. Since $\Sigma(G, G / M A N)$ is compact and contains a dense image of $G$, it means the same thing to say that there is $z \in \Sigma(G, G / M A N)$ with $z(x)=z(y)([10]$, Theorem IV(3.22)). For the proof of Theorem 2.1, see the references cited above or [10, $\mathrm{VI}(6.5)$.

Theorem 2.1 has the following corollary which appears in [10, IV(6.8)3.

Corollary 2.2. The flow $(A N, G / M A N)$ is proximal.

Here is a sketch of its proof. Given $x, y \in G / M A N$, there is a net $\left(k_{i} a_{i} n_{i}\right)$ in $K A N=G$ with $\lim _{i} k_{i} a_{i} n_{i}(x)=\lim _{i} k_{i} a_{i} n_{i}(y)$. Compactness allows us to assume $k_{i} \rightarrow k \in K$ and $a_{i} n_{i} \rightarrow z \in \Sigma(A N, G / M A N)$. Ellis's joint continuity theorem ([2], Appendix B) tells us $k_{i} a_{i} n_{i} \rightarrow k z$, whence $k z(x)=k z(y)$ and as $k$ is invertible, $z(x)=z(y)$.

The left action of $M$ on $G / A N \cong K$ is just the group multiplication of elements of $K$ on the left by elements of $M$. However, $M$ also has a continuous right action on $G / A N$ which is essentially just multiplication on the right by elements of $M$ : for $g \in G, m \in M$ we have

$$
g A N m=g m A N
$$

since $M$ is in the normalizer of $A N$. Notice that this formula shows that the right action of $M$ commutes with the (left) action of $G$; as a consequence, it also commutes with the (left) action of $\Sigma(G, G / A N)$. When $G / A N$ is identified with $K$, the right action is, of course, just right multiplication:

$$
(k, m) \mapsto k m, K \times M \rightarrow K,
$$

and we have

$$
(y(k)) m=y(k m), \quad y \in \Sigma(G, G / A N), k \in K, m \in M .
$$

We next see that the flows $(A N, G / A N)$ and $(A N, G / M A N)$ have fixed points. 
Proposition 2.3. The fixed points of $(A N, G / A N)$ are the cosets $m A N$ with $m \in$ $M$ (or when $G / A N$ is identified with $K$, simply the elements of $M$ ). The flow $(A N, G / M A N)$ has exactly one fixed point, the coset MAN.

Proof. For $m \in M$ and $a n \in A N$ we find $a n(m A N)=a n A N m=A N m=m A N$, so $m A N$ is a fixed point. Conversely, let $k \in K \cong G / A N$ be fixed by $A N$. Then ank $A N=k A N$ for all $a n \in A N$. In particular ank $\in k A N$, whence $k^{-1} A N k \subseteq A N$. Now $\left\{k^{\prime} \in K: k^{\prime-1} A N k^{\prime} \subseteq A N\right\}$ is a closed subsemigroup of the compact group $K$, and therefore it contains $k^{-1}$ whenever it contains $k$ (see [3], Theorem $1.10 \mathrm{ff}$.). Therefore $k^{-1} A N k=A N$, so that $k$ is a normalizer of $A N$ lying in $K$, that is $k \in M$.

It follows that the coset $M A N$ is a fixed point of $(A N, G / M A N)$. Obviously a proximal flow can have no more than one fixed point, so our final assertion follows from Theorem 2.1.

We now begin to consider the enveloping semigroups of our flows.

Proposition 2.4. The enveloping semigroup $\Sigma(A N, G / M A N)$ has a (semigroup) zero.

Proof. We know (Proposition 2.3) that the $\operatorname{coset} x_{0}=M A N$ is a fixed point for the flow, and therefore a fixed point for all maps in $\Sigma(A N, G / M A N)$. We show that the map $z_{0}: G / M A N \rightarrow G / M A N$ defined by $z_{0}(x)=x_{0}(x \in G / M A N)$ belongs to $\Sigma(A N, G / M A N)$; it is then obvious that it is a semigroup zero. By the compactness of $\Sigma(A N, G / M A N)$ it is enough to show that for each finite subset $\left\{x_{1}, \ldots, x_{n}\right\}$ of $G / M A N$ there is $z \in \Sigma(A N, G / M A N)$ with $z\left(x_{i}\right)=x_{0}(1 \leq i \leq n)$. By proximality (Corollary 2.2) we can find $z_{1} \in \Sigma(A N, G / M A N)$ with $z_{1}\left(x_{1}\right)=z_{1}\left(x_{0}\right)=x_{0}$. We can then find $z_{2} \in \Sigma(A N, G / M A N)$ with $z_{2}\left(z_{1}\left(x_{2}\right)\right)=z_{2}\left(x_{0}\right)=x_{0}$; notice also that $z_{2}\left(z_{1}\left(x_{1}\right)\right)=z_{2}\left(x_{0}\right)=x_{0}$. Continuing in this way we find $z_{1}, \ldots, z_{n}$ with $\left(z_{n} \ldots z_{1}\right)\left(x_{i}\right)=x_{0}, 1 \leq i \leq n$.

This zero is obviously the (unique) minimal left ideal of $\Sigma(A N, G / M A N)$. We next find the minimal left ideals of $\Sigma(A N, G / A N)$.

Proposition 2.5. The set

$$
I=\{w \in \Sigma(A N, G / A N): w(G / A N) \subseteq\{m A N: m \in M\}\}
$$

is non-empty and is the set of right zeros of $\Sigma(A N, G / A N)$. For each $w \in I$, the singleton $\{w\}$ is a minimal left ideal in $\Sigma(A N, G / A N)$, and all minimal left ideals are of this form.

Proof. The natural map $\varphi: G / A N \rightarrow G / M A N, g A N \mapsto g M A N$, maps the orbits of the right action of $M$ on $G / A N$ to points, and so it is a flow homomorphism $(A N, G / A N) \rightarrow(A N, G / M A N)$. It therefore lifts to a continuous semigroup homomorphism $\varphi_{\Sigma}: \Sigma(A N, G / A N) \rightarrow \Sigma(A N, G / M A N)$.

Take a net $\left(a_{i} n_{i}\right)$ in $A N$ which converges in $\Sigma(A N, G / M A N)$ to its zero $z_{0}$. Then there is a subnet $\left(a_{j} n_{j}\right)$ of $\left(a_{i} n_{i}\right)$ which converges in $\Sigma(A N, G / A N)$ to some element, say $w$. For any $k \in G / A N$,

$$
\varphi(w(k))=\lim _{j} \varphi\left(a_{j} n_{j}(k)\right)=\lim _{j} \varphi_{\Sigma}\left(a_{j} n_{j}\right)(\varphi(k))=z_{0}(\varphi(k))=M A N .
$$

Thus $I \neq \emptyset$. Since each element $m A N$ is fixed in $G / A N$ for the action of $A N$, and so also for the action of $\Sigma(A N, G / A N)$, we see that each element of $I$ is a right zero in $\Sigma(A N, G / A N)$, and so forms a minimal left ideal. 
If $w$ is a right zero, then for $k \in K, w(k A N)$ is fixed under the action of $A N$ and therefore $w(k A N) \in\{m A N: m \in M\}$ by Proposition 2.3.

In our next result we consider $\Sigma_{2}=\Sigma(A N, G / A N)$ as a compact subsemigroup of $\Sigma_{1}=\Sigma(G, G / A N)$.

N.B. From now on we identify the quotient space $G / A N$ with $K$. The reader must bear in mind that $K$ also appears as a subgroup of $G$ and of $\Sigma_{1}$; for example, in (1) of Lemma $2.8 E_{w}$ is defined as a subset of $K=G / A N$, and then appears in (2) as a subset of $K \subset \Sigma_{1}$.

Proposition 2.6. $\Sigma(G, K)=K \Sigma(A N, K)$ (that is, $\left.\Sigma_{1}=K \Sigma_{2}\right)$.

Proof. If $z \in \Sigma_{1}$, there is a net $\left(k_{i} a_{i} n_{i}\right)$ in $K A N=G$ with $k_{i} a_{i} n_{i} \rightarrow z$. Compactness allows us to assume that $k_{i} \rightarrow k$ in $K$ and $a_{i} n_{i} \rightarrow y$ in $\Sigma_{2}$. Ellis's Theorem (e.g., 1. Appendix B]) guarantees the joint continuity of the map $K \times \Sigma_{1} \rightarrow \Sigma_{1}$ and we conclude that $z=\lim _{i} k_{i} a_{i} n_{i}=k y$.

Proposition 2.6 suggests that, since $K$ is a group, it would be reasonable to expect that if $w$ was a right zero of $\Sigma_{2}$ (so that $\{w\}$ was a minimal left ideal of $\Sigma_{2}$ ), then $K w$ would be a minimal left ideal in $\Sigma_{1}$ and would be a group algebraically. The first of these suggestions turns out to be correct but the second is false, as we shall see.

Lemma 2.7. Let $w$ be a right zero in $\Sigma_{2}$.

(1) Then $K w$ is a closed left ideal in $\Sigma_{1}$ and is homeomorphic to $K$.

(2) If $w^{\prime}$ is another right zero in $\Sigma_{2}$, then $K w \cap K w^{\prime}=\emptyset$.

Proof. Let $g \in G, k \in K$. Then $g k \in G=K A N$, so $g k=k^{\prime} a^{\prime} n^{\prime}$ for some $k^{\prime}, a^{\prime}, n^{\prime}$. Thus $g k w=k^{\prime} a^{\prime} n^{\prime} w=k^{\prime} w \in K w$. Since $K w$ is closed and $G$ is dense in $\Sigma_{1}$ it follows that $K w$ is a left ideal. The map $\theta: k \mapsto k w, K \rightarrow K w \subset \Sigma_{1}$ is clearly continuous, as is $k \mapsto k w\left(k^{\prime}\right)$ of $K$ onto $K$ (for any fixed $k^{\prime}$ ), and the latter map is also $1-1$; this proves $(1)$.

If $k w=k^{\prime} w^{\prime}$ for $k, k^{\prime} \in K$, then $k w=k w^{2}=k^{\prime} w^{\prime} w=k^{\prime} w$ (Proposition 2.5), and so $k=k^{\prime}$ by (1), and $w=k^{-1} k w=k^{-1} k w^{\prime}=w^{\prime}$; this proves (2).

Lemma 2.8. Let e be the identity of $M$, and let $w$ be a right zero in $\Sigma_{2}$. Set

$$
E_{w}=\{k \in K: w(k)=e\} .
$$

(1) For any $m \in M, E_{w} m=\{k \in K: w(k)=m\}$, and $K$ is the disjoint union

$$
K=\bigcup_{m \in M} E_{w} m=E_{w} M
$$

(2) The idempotents in $K w$ are given by $E_{w} w=\left\{k w: k \in E_{w}\right\}$ and form a left zero semigroup.

(3) The map $\psi: E_{w} \times M \rightarrow K w$ given by $\psi(k, m)=k m w$ is bijective and is a homomorphism when $E_{w} \times M$ is given the left group product $\left(k_{1}, m_{1}\right)\left(k_{2}, m_{2}\right)=$ $\left(k_{1}, m_{1} m_{2}\right)$.

(4) The maximal subgroups of $K w$ have the form $k M w$ for $k \in E_{w}$; they are closed and isomorphic (topologically and algebraically) to $M$.

Proof. (1) That $w\left(k^{\prime}\right)=m$ if and only if $k^{\prime} \in E_{w} m$ follows from the fact that the right action of $M$ commutes with the action of $\Sigma_{2}$ - see $(*)$ before Proposition 2.3 - so that $\left(w\left(k^{\prime}\right)\right) m^{-1}=w\left(k^{\prime} m^{-1}\right)$. Thus (1) is established. 
(2) Let $m \in M$, the range of $w$. Then since $k w\left(E_{w} m\right)=k m$, we have

$$
k w\left(k w\left(E_{w} m\right)\right)=k w(k m),
$$

which is equal to $k m=k w\left(E_{w} m\right)$ if and only if $k \in E_{w}$, by (1). This proves the first claim of (2), and the proof of the second claim of (2) is similar.

(3) From (1) and Lemma 2.7, $\psi$ is obviously bijective. If $k_{1}, k_{2} \in E_{w}$ and $m_{1}, m_{2} \in M$, then arguing as in (2) we have $k_{2} m_{2} w\left(E_{w} m\right)=k_{2} m_{2} m$ which yields

$$
\left(k_{1} m_{1} w\right)\left(k_{2} m_{2} w\right)=k_{1} m_{1} m_{2} w ;
$$

but this is just $\psi\left(k_{1}, m_{1}\right) \psi\left(k_{2}, m_{2}\right)=\psi\left(k_{1}, m_{1} m_{2}\right)=\psi\left(\left(k_{1}, m_{1}\right)\left(k_{2}, m_{2}\right)\right)$ as required.

(4) From (3) the maximal subgroups of $K w$ are of the form $\psi(k, M)=k M w$ and they are isomorphic to $M$. They are homeomorphic with $M$ from Lemma 2.7 (1).

Proof of Theorem 1.1. We must show that the left ideal $K w$ (Lemma 2.7) is minimal. But this is immediate from Lemma 2.8 (3), since any semigroup of the form $E \times \Gamma$ where $E$ is a left zero semigroup and $\Gamma$ is a group contains no proper left ideals.

Corollary 2.9. If $M$ is finite, the set of idempotents in each minimal left ideal of $\Sigma_{1}$ is not closed.

Proof. The sets $E_{w} m(m \in M)$ form a finite partition of $K$ under our hypotheses, and if one of these sets is closed they all are. But this means that each is also open (in $K$ ). Since $K$ is connected, this cannot happen. The map $K \rightarrow K w$ is a homeomorphism (Lemma $2.7(1)$ ), so the set $E_{w} w$ of idempotents in the minimal left ideal $K w_{0}$ is not closed.

Finally in this section we look at the semigroup $\Sigma(G, G / M A N)$. We are able to say more about its ideal structure. We want (a variant of) Proposition 2.6 in this context. To state it properly, we must recognize that the map $G \rightarrow \Sigma(G, G / M A N)$ might not be $1-1$ on $K$. However, each $k \in K$ does act on $\Sigma(G, G / M A N)$; for $s \in \Sigma(G, G / M A N)$ and $x \in G / M A N$ we write $k(s)(x)=k(s(x))$.

Proposition 2.10. We have $\Sigma(G, G / M A N)=K(\Sigma(A N, G / M A N))$. If $z_{0}$ is the zero of $\Sigma(A N, G / M A N)$, then the minimal right ideals of $\Sigma(G, G / M A N)$ are the singletons of the form $\left\{k\left(z_{0}\right)\right\}$ for $k \in K$. The minimal ideal of $\Sigma(G, G / M A N)$ is $K\left(z_{0}\right)$. It is a left zero semigroup and is homeomorphic to $K / M$.

Proof. As in the proof of Proposition 2.6 we see that any element of $\Sigma(G, G / M A N)$ is of the form $k(s)(k \in K, s \in \Sigma(A N, G / M A N))$.

For each $s \in \Sigma(G, G / M A N), x \in G / M A N$ we have $\left(z_{0} s\right)(x)=z_{0}(s(x))=x_{0}$ (with the notation of Proposition 2.4). Therefore $z_{0}$ is a left zero in $\Sigma(G, G / M A N)$. Thus $\left\{z_{0}\right\}$ is a minimal right ideal, and hence all minimal right ideals are singletons. From the beginning of this proof they will be of the form $\left\{k\left(z_{0}\right)\right\}$ for $k \in K$. The union of all these right ideals, that is $K\left(z_{0}\right)$, is the unique minimal left ideal and also the minimal two-sided ideal of $\Sigma(G, G / M A N) . K\left(z_{0}\right)$ is a continuous image of $K$ and so compact, and the map $K \rightarrow K\left(z_{0}\right)$ has kernel $\left\{k: k\left(z_{0}\right)=z_{0}\right\}=\{k:$ $k M A N=M A N\}=M$, so that $K\left(z_{0}\right)$ is homeomorphic with $K / M$. 


\section{The examples $\operatorname{SL}(2, \mathbb{C})$ and $\operatorname{SL}(2, \mathbb{R})$}

In this section in order to illuminate the general theory of $\S 2$ we discuss the enveloping semigroup $\Sigma_{1}=\Sigma(G, K)=\Sigma(G, G / A N)$ when $G=\operatorname{SL}(2, \mathbb{C})$ and $\mathrm{SL}(2, \mathbb{R})$. It is remarkable that fairly complete descriptions of these semigroups can be given and that they are in many ways uncomplicated. One point to notice is that for $\mathrm{SL}(2, \mathbb{C})$, even though the group $M$ is not finite (so that Corollary 2.9 does not apply), the set of idempotents in a minimal left ideal of the enveloping semigroup is not closed.

3.1. We begin by describing a particular compact semigroup $\mathcal{S}$ (which will turn out to be isomorphic with $\Sigma_{2} \backslash A N=\Sigma(A N, K) \backslash A N$ for $\left.\operatorname{SL}(2, \mathbb{C})\right)$.

We denote by $\overline{\mathbb{C}}=\left\{r e^{i \theta}: 0 \leq r \leq \infty,-\pi<\theta \leq \pi\right\}$ a compactified complex plane; as usual all points $0 e^{i \theta}$ are equal to 0 , but the boundary $\partial \overline{\mathbb{C}}=\overline{\mathbb{C}} \backslash \mathbb{C}=$ $\left\{\infty e^{i \theta}:-\pi<\theta \leq \pi\right\}$ is a 'circle at infinity'. The topology on $\overline{\mathbb{C}}$ is such that $r e^{i \theta} \mapsto r e^{i \theta} /(1+r)$ is a homeomorphism onto the closed unit disc.

The semigroup $\mathcal{S}$ will consist of two families of maps of $\overline{\mathbb{C}}$ into itself,

$$
\text { (i) }\left\{v_{b}: b \in \overline{\mathbb{C}}\right\}, \quad \text { (ii) }\left\{w_{a, b}: a \in \mathbb{C}, b \in \overline{\mathbb{C}}\right\} \text {; }
$$

these are defined by the requirement that all of them leave the points of $\partial \overline{\mathbb{C}}$ fixed and, in addition, for $z \in \mathbb{C}$,

$$
\text { (i) } v_{b}(z)=b, \quad \text { (ii) } w_{a, b}(z)= \begin{cases}\infty e^{i \arg (z-a)} & \text { if } z \neq a \\ b & \text { if } z=a\end{cases}
$$

More intuitively, when $z \neq a, w_{a, b}(z)$ is the point at infinity at the end of the ray drawn through $z$ from $a$. It is easy to see that these maps do form a semigroup under composition. The right zeros of this semigroup are the elements with range in $\partial \overline{\mathbb{C}}$, that is the maps $v_{b}$ and $w_{a, b}$ with $b \in \partial \overline{\mathbb{C}}$. Together these elements form the minimal ideal.

We give $\mathcal{S}$ the topology of pointwise convergence on $\overline{\mathbb{C}}$. For some sequences convergence is straightforward: if $a_{n} \rightarrow a$ and $b_{n} \rightarrow b$ in $\overline{\mathbb{C}}$, then $v_{a_{n}} \rightarrow v_{a}$ and for any $a \in \mathbb{C}, w_{a, b_{n}} \rightarrow w_{a, b}$. For other sequences the situation is more complicated. For example, let $a_{n} \rightarrow a$ in $\mathbb{C}$ with $a_{n} \neq a$ for all $n$ and $\lim _{n} \arg \left(a-a_{n}\right)=\phi$. Then, for any $b \in \overline{\mathbb{C}}, w_{a_{n}, b} \rightarrow w_{a, \infty e^{i \phi}}$. An interesting aspect of this case is that the limit function does not depend on $b$ in any way. This feature is reminiscent of others which occur in the context of minimal ideals in compact semigroups (see for example, Example 1 of [1]). We shall not go further into the details of the topology on $\mathcal{S}$, though we should mention that it is compact.

3.2. We next describe the action of $A N$ on $K=G / A N$ for $G=\mathrm{SL}(2, \mathbb{C})$ and find $\Sigma_{2}$ in this case. The expression of $g \in \mathrm{SL}(2, \mathbb{C})$ in the form $k a n$ is given by

$$
k=\left(\begin{array}{cc}
s & -\bar{t} \\
t & \bar{s}
\end{array}\right), \quad a=\left(\begin{array}{cc}
\varepsilon & 0 \\
0 & \varepsilon^{-1}
\end{array}\right), \quad n=\left(\begin{array}{ll}
1 & \xi \\
0 & 1
\end{array}\right)
$$

where $|s|^{2}+|t|^{2}=1(s, t \in \mathbb{C}), \varepsilon>0$ and $\xi \in \mathbb{C}$. We shall use the abbreviations $k=(s, t), a=(\varepsilon)$ and $n=(\xi)$ for these matrices. The normalizer $M$ of $A N$ in $K$ consists of the diagonal elements of $K$, that is $(s, 0)$ with $|s|=1$. 
It will be convenient to write $(s, t) \in K$ as $\left(s, \rho e^{i \alpha}\right)$; then $|s|^{2}+\rho^{2}=1$ so that $\rho \geq 0$ is determined by $s$. For $-\pi<\alpha \leq \pi$, we shall put

$$
S_{\alpha}=\left\{\left(s, \rho e^{i \alpha}\right):|s|<1,|s|^{2}+\rho^{2}=1\right\}
$$

$\overline{S_{\alpha}}$ for its closure (so including points with $|s|=1$ ) and $\partial \overline{S_{\alpha}}=\overline{S_{\alpha}} \backslash S_{\alpha}$. Note that for $\alpha \neq \gamma, \overline{S_{\alpha}} \cap \overline{S_{\gamma}}=\{(s, 0):|s|=1\}=\partial \overline{S_{\alpha}}$, and that this is precisely $M$. Thus $\partial \overline{S_{\alpha}}$ is independent of $\alpha$.

If we denote the image of $\left(s, \rho e^{i \alpha}\right)$ under the action of $(\varepsilon)(\xi) \in A N$ by $\left(s^{\prime}, \rho^{\prime} e^{i \alpha^{\prime}}\right)$ we find (using the Gram-Schmidt process as on page 117 of [5]) that

$$
\begin{aligned}
s^{\prime} & =\frac{\varepsilon^{2} s+\varepsilon^{2} \xi \rho e^{i \alpha}}{\sqrt{\left|\varepsilon^{2} s+\varepsilon^{2} \xi \rho e^{i \alpha}\right|^{2}+|\rho|^{2}}}, \\
\rho^{\prime} e^{i \alpha^{\prime}} & =\frac{\rho e^{i \alpha}}{\sqrt{\left|\varepsilon^{2} s+\varepsilon^{2} \xi \rho e^{i \alpha}\right|^{2}+|\rho|^{2}}} .
\end{aligned}
$$

Obviously $\alpha=\alpha^{\prime}$ so that $S_{\alpha}$ is an invariant subset, and the formulas show that $S_{\alpha}$ is even a minimal invariant set. Since the points of $M=\partial \overline{S_{\alpha}}$ are invariant, we see that $\overline{S_{\alpha}}$ is a compact invariant subset.

The image of $S_{\alpha}$ under the right action of $m_{\gamma}=\left(e^{-i \gamma}, 0\right) \in M$ is $S_{\alpha+\gamma}$. We know that this action of $M$ commutes with the action of $A N$ (see after Corollary 2.2) so that the action of $A N$ on any one disc $S_{\alpha}$ determines the flow. Specifically if we fix $\alpha=0$ and take $s \in S_{\gamma}$ we find $((\varepsilon)(\xi))(s)=((\varepsilon)(\xi))\left(s m_{\gamma}^{-1}\right) m_{\gamma}$. The compact semigroup $\Sigma_{2}$ is therefore isomorphic to $\Sigma\left(A N, \overline{S_{0}}\right)$. (We take $\overline{S_{0}}$ rather than $S_{0}$ because we need a compact phase space. Recall that the points of $\partial \overline{S_{0}}$ are fixed for the action of $A N$, though not for the action of $M$.)

Now $\overline{S_{0}}=\left\{(s, \rho):|s|^{2}+\rho^{2}=1\right\}$. The map $(s, \rho) \mapsto s \mapsto s / \sqrt{1-|s|^{2}}$ is a homeomorphism of $\overline{S_{0}}$ with the space $\overline{\mathbb{C}}$ of $\S 3.1$. Notice that $\partial \overline{S_{0}}=M$ maps to $\partial \overline{\mathbb{C}}$, and that the identity of $M$ goes to $\infty e^{i 0}=\infty 1 \in \partial \overline{\mathbb{C}}$.

The action on $\overline{\mathbb{C}}$ which is equivalent to the action of $(\varepsilon)(\xi)$ on $\overline{S_{0}}$ can be read from the formula for the action on $S_{0}$ given earlier: if $s \in S_{0}$ corresponds to $z \in \mathbb{C}$, then $(\varepsilon)(\xi)$ sends $z$ to $z^{\prime}=\varepsilon^{2} z+\varepsilon^{2} \xi=\varepsilon^{2}(z+\xi)$ and it leaves all $z \in \partial \overline{\mathbb{C}}$ fixed. This is (an extension to $\overline{\mathbb{C}}$ of) a standard affine action on $\mathbb{C}$.

It is fairly straightforward to find all the elements of $\Sigma(A N, \overline{\mathbb{C}})$. The possible cluster points of $\varepsilon^{2}$ are in $[0, \infty]$; the possible cluster points of $\varepsilon^{2} \xi$ are either in $\mathbb{C}$ or in $\partial \overline{\mathbb{C}}$. When we are looking for elements of $\Sigma(A N, \overline{\mathbb{C}})$ we may assume that all nets involving $\varepsilon^{2}$ or $\varepsilon^{2} \xi$ converge in $[0, \infty]$ or $\overline{\mathbb{C}}$. In nearly all cases we find limit maps of the form $v_{b}$ (see $\S 3.1$ ), and we can obtain all $v_{b}$ with $b \in \overline{\mathbb{C}}$. The main case in which the result is different occurs when $\varepsilon^{2} \rightarrow \infty$ and $\varepsilon^{2} \xi \rightarrow \infty e^{i \phi} \in \partial \overline{\mathbb{C}}$. We need also to assume that $\varepsilon^{2} \xi / \varepsilon^{2}=\xi$ converges to $\zeta_{1} \in \mathbb{C}$ say (if $\zeta_{1} \in \partial \overline{\mathbb{C}}$ we again have a limit of the form $v_{b}$ ), and we find the limit map is $w_{-\zeta_{1}, b}$ for some $b \in \overline{\mathbb{C}}$. Again all possible maps of this form can be obtained.

We conclude that the semigroup $\Sigma_{2}$ is isomorphic with $\Sigma(A N, \overline{\mathbb{C}})=A N \cup \mathcal{S}$.

The size of the semigroup $\mathcal{S}$ which is adjoined to $A N$ to form this enveloping semigroup is rather small, and consists of a restricted class of mappings. The image of $\mathbb{C}$ under an element of $\mathcal{S}$ is either a singleton or a singleton together with $\partial \overline{\mathbb{C}}$. 
3.3. We now consider the structure of the minimal left ideals in $\Sigma_{1}=K \Sigma_{2}$. All are isomorphic and of the form $K w$ where $w$ is a right zero of $\Sigma_{2}$. As in $\S 2$, we write $K=M \cup \bigcup_{\pi<\alpha \leq \pi} S_{\alpha}$. The right action of $M$ permutes the $S_{\alpha}$ 's.

In $\S 3.2$, what precisely was determined was $\Sigma\left(A N, \overline{S_{0}}\right)$. A map $v \in \Sigma\left(A N, \overline{S_{0}}\right)$ extends uniquely to $\tilde{v} \in \Sigma_{2}$ through the formula

$$
\tilde{v}(k)=\left(v\left(k m_{-\alpha}\right)\right) m_{\alpha} \quad \text { if } k \in S_{\alpha}=S_{0} m_{\alpha}
$$

(of course $\tilde{v}(k)=k$ for all $k \in M$ ). This formula means that the right zeros of $\Sigma_{2}$ are the maps $\tilde{v}_{b}$ and $\tilde{w}_{a, b}$ with $b \in M$.

We now determine some of the idempotents in $\Sigma_{1}$. According to Lemma 2.8, $k \tilde{v}_{m_{\alpha}}$ is an idempotent iff $\tilde{v}_{m_{\alpha}}(k)=1$ (the identity of $M$ ). We consider two cases. First, if $k \in M$, then $\tilde{v}_{m_{\alpha}}(k)=k$, so $k \tilde{v}_{m_{\alpha}}$ is an idempotent if and only if $k=1$, that is $k \tilde{v}_{m_{\alpha}}=\tilde{v}_{m_{\alpha}}$. Secondly when $k \in S_{\gamma}, \tilde{v}_{m_{\alpha}}(k)=\left(v_{m_{\alpha}}\left(k m_{-\gamma}\right)\right) m_{\gamma}=m_{\alpha} m_{\gamma}$, so $k \tilde{v}_{m_{\alpha}}$ is an idempotent if and only if $\gamma=-\alpha$; this means all elements $k \tilde{v}_{m_{\alpha}}$ with $k \in S_{-\alpha}$ are idempotents. Thus $E_{\tilde{v}_{m_{\alpha}}}=S_{-\alpha} \cup\{1\}$, and $K \tilde{v}_{m_{\alpha}}=E_{\tilde{v}_{m_{\alpha}}} M$ (which is isomorphic with $\left.E_{\tilde{v}_{m_{\alpha}}} \times M\right)$.

An immediate consequence of the above formulation is that the idempotents in $K \tilde{v}_{m_{\alpha}}$ do not form a closed set. Indeed the closure $\bar{S}_{-\alpha} \tilde{v}_{m_{\alpha}}$ of $S_{-\alpha} \tilde{v}_{m_{\alpha}}$ contains $M \tilde{v}_{m_{\alpha}}$, but the only idempotent member of $M \tilde{v}_{m_{\alpha}}$ is $\tilde{v}_{m_{\alpha}}$ itself.

3.4. The description of the enveloping semigroup for $G=\mathrm{SL}(2, \mathbb{R})$ is much simpler. First, $K$ is just the circle $\mathbb{T}$, which we identify with $[0,2 \pi)$. The subgroup $M$ is $\{0, \pi\}$. The action of an element of $K$ on $\mathbb{T}$ is just a rotation. The elements of $\Sigma(G, K) \backslash G$ can be described as follows. Take $0 \leq \phi<\pi$. Partition $\mathbb{T}$ into $\{\phi\}$, $(\phi, \phi+\pi),\{\phi+\pi\}$ and $(\phi+\pi, \phi+2 \pi)$. Take $\psi_{1}, \psi_{2} \in[0,2 \pi)$ and define maps $U_{\phi, \psi_{1}, \psi_{2}}$ by

$$
\begin{array}{cc}
U_{\phi, \psi_{1}, \psi_{2}}(\phi)=\psi_{1}, & U_{\phi, \psi_{1}, \psi_{2}}(\phi+\pi)=\psi_{1}+\pi \\
U_{\phi, \psi_{1}, \psi_{2}}((\phi, \phi+\pi))=\left\{\psi_{2}\right\}, & U_{\phi, \psi_{1}, \psi_{2}}((\phi+\pi, \phi+2 \pi))=\left\{\psi_{2}+\pi\right\} .
\end{array}
$$

These maps lie in the minimal ideal when their images consist of just two points (so either $\psi_{1}=\psi_{2}$ or $\psi_{1}=\psi_{2}+\pi$ ). In the latter situation, $U_{\phi, \psi, \psi}$ is idempotent when $\psi \in[\phi, \phi+\pi)$. Otherwise, $U_{\phi, \psi, \psi}([\phi, \phi+\pi)) \subseteq[\phi+\pi, \phi+2 \pi)$ whence $U_{\phi, \psi, \psi}$ is not idempotent but $U_{\phi, \psi, \psi}^{2}$ is. That these idempotents do not form a closed set is seen by letting $\psi \nearrow \phi+\pi$ (as well as from Corollary 2.9).

\section{REFERENCES}

1. J W Baker and P Milnes, The ideal structure of the Stone-Čech compactification of a group, Math Proc Camb Phil Soc 82 (1977), 401-409. MR 57:509

2. J F Berglund, H D Junghenn and P Milnes, Analysis on Semigroups, Wiley, New York, 1989. MR 91b:43001

3. J H Carruth, J A Hildebrandt and R J Koch, The Theory of Topological Semigroups I, Dekker, New York, 1983. MR 84g:22002

4. S Glasner, Proximal Flows, Lecture Notes in Mathematics 517, Springer, Berlin, 1976. MR 57:13890

5. A W Knapp, Representation Theory of Semisimple Groups, Princeton University Press, Princeton, 1986. MR 87j:22022

6. A W Knapp, Lie Groups Beyond an Introduction, Birkhäuser, Boston, 1996. MR 98b:22002

7. A T Lau, P Milnes and J Pym, Compactifications of locally compact groups and closed subgroups, Trans Amer Math Soc 329 (1992), 97-115. MR 92e:43004]

8. A T Lau, P Milnes and J Pym, Flows on invariant subsets and compactifications of a locally compact group, Coll Math 78 (1998), 267-281. MR 2000e:43005 
9. C Moore, Compactifications of symmetric spaces, Amer J Math 86 (1964), 201-218. MR 28:5146

10. J de Vries, Elements of Topological Dynamics, Kluwer, The Netherlands, 1993. MR 94m:54098

11. G Warner, Harmonic Analysis on Semisimple Lie Groups I, Springer, Berlin, 1972. MR 58:16979

Department of Mathematics, BoĞaziçi Üniversitesi, 80815 Bebek, İstanbul, Turkey

E-mail address: budakt@boun.edu.tr

Department of Mathematics, BoĞaziçi Üniversitesi, 80815 Bebek, İstanbul, Turkey

E-mail address: isikn@boun.edu.tr

Department of Mathematics, University of Western Ontario, London, Ontario, Canada N6A 5B7

E-mail address: milnes@uwo.ca

Department of Pure Mathematics, University of Sheffield, S3 7RH, England

E-mail address: j.pym@shef .ac.uk 